\section{Esquizofrenia y su asociación con enfermedades médicas crónicas}

\author{
GRICEL ORELLANA ${ }^{1, \mathrm{a}}$, MATÍAS RODRÍGUEZ ${ }^{2, \mathrm{~b}}$, \\ NICOLÁS GONZÁLEZ ${ }^{2, \mathrm{~b}}$, EDUARDO DURÁN ${ }^{2, \mathrm{c}}$
}

\section{The association of schizophrenia with chronic non transmissible diseases}

The life expectancy of patients with schizophrenia (SCH) is 11 to 20 years less than the general population. There is an association between SCH and various diseases and chronic conditions, highlighting the cardio-metabolic diseases. This association has been attributed to the use of antipsychotics, however, evidence has also shown intrinsic susceptibility of schizophrenic patients the development of chronic conditions. This review aims to update knowledge about chronic conditions such as cardiometabolic risk and sleep, bone and kidney disorders related to SCH. These patients have a high prevalence of risk behaviors, including smoking and poor diet. They have a worse metabolic profile than the general population and a greater likelihood of developing metabolic syndrome, diabetes and cardiovascular disease. SCH has also been associated with other chronic diseases such as osteoporosis and chronic kidney disease. The high prevalence of these comorbidities in schizophrenic population is not explained solely by the antipsychotic treatment, therefore intrinsic mechanisms associated to SCH are postulated to be associated with these conditions. This new information requires a change in the multidisciplinary medical approach for the study and management of schizophrenic patients.

(Rev Med Chile 2017; 145: 1047-1053)

Key words: Antipsychotic Agents; Metabolic Syndrome X; Schizophrenia.

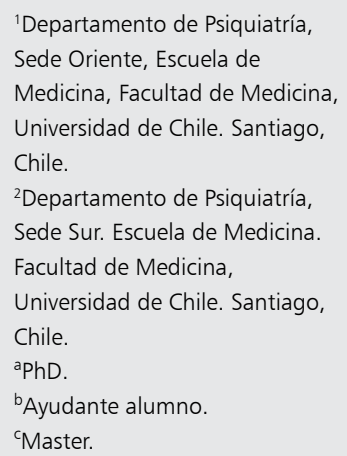

Recibido el 27 de mayo de 2016, aceptado el 17 de noviembre de 2016.

Correspondencia a: Gricel Paulina Orellana Vidal Departamento de Psiquiatría Oriente, Av. Salvador 486 Providencia, Santiago Chile. gricel.orellana@gmail.com

\section{I} a esquizofrenia (EQZ) afecta a $1 \%$ de la población mundial. Es una enfermedad psiquiátrica grave y crónica que afecta significativamente la funcionalidad y calidad de vida del individuo.

Se caracteriza por síntomas positivos (alucinaciones, delirio, síntomas catatónicos y discurso desorganizado), negativos (aplanamiento afectivo y anhedonia) y disfunciones cognitivas (alteración de atención, memoria y funciones ejecutivas) ${ }^{1}$. Además, hay perturbaciones fisiológicas severas que aumentan la gravedad de la EQZ, de hecho, se evidencian alteraciones endocrinológicas, del sueño, cardiovasculares y metabólicas con alteración del peso, regulación de glicemia, metabolismo lipídico y presión arterial, cuyos mecanismos no se conocen del todo ${ }^{2}$.
La expectativa de vida de los pacientes con EQZ es entre 11 y 20 años menor que la población general y la mortalidad cardiovascular ha aumentado en este grupo de pacientes, en contraste a la población general ${ }^{3}$.

El tratamiento antipsicótico siempre se ha asociado a estas alteraciones cardiometabólicas, sin embargo, la evidencia muestra que pacientes que nunca han recibido tratamiento también presentan estas patologías concomitantes ${ }^{4}$, además que muchas de ellas se encuentran desde el primer episodio psicótico, por ende, se postula que la EQZ per se estaría relacionada con esta alteración ${ }^{5,6}$.

Este trabajo pretende actualizar los conocimientos con respecto a comorbilidades médicas crónicas asociadas a la EQZ y ayudar así a optimizar el manejo clínico de estos pacientes. 


\section{Metodología}

Se llevó a cabo una investigación de 42 artículos disponibles, escritos en idioma inglés, tanto de investigación como de revisión, incluyéndose aquellos que relacionaban la presencia de EQZ con comorbilidad médica y excluyéndose aquellos anteriores a 1999, por medio de las bases de datos Medline/PubMed y sitios web de tipo científicos, abarcando desde el año 2000 hasta el año 2015.

\section{Resultados}

\section{Hábitos}

Los pacientes con EQZ tienen mayor prevalencia de tabaquismo que la población general ${ }^{7}$, con un promedio de 4 cigarrillos por día ${ }^{8}$. Se ha postulado que el tabaquismo puede tener un efecto sobre el sistema dopaminérgico mesolímbico, aumentando la liberación de dopamina a la corteza prefrontal y aliviando así síntomas positivos y negativos de manera parcial, lo que explicaría en algún grado los niveles de tabaquismo de los pacientes con EQZ ${ }^{9}$. Relacionado a lo anterior, cerca de $50 \%$ de los pacientes con EQZ presentan trastorno por uso de sustancias, explicado por las mismas vías descritas. Este trastorno está implicado en un peor resultado terapéutico, pudiendo llegar a aumentar los síntomas negativos y las disfunciones cognitivas ${ }^{10}$.

Con respecto al peso, $50 \%$ de estos pacientes son obesos y menos de $20 \%$ realiza algún tipo de actividad física regular. Además, los pacientes con EQZ consumen más calorías que la población general, prefiriendo azúcares y grasas saturadas por sobre frutas y verduras ${ }^{11}$. Estos son factores modificables que contribuyen a las alteraciones cardio-metabólicas evidenciadas en los pacientes con EQZ, por lo que se deben pesquisar y manejar para controlar las patologías asociadas.

El aumento de peso durante el tratamiento de la EQZ, tanto agudo como de mantención, es un efecto bien establecido y demostrado de los antipsicóticos ${ }^{12}$. El principal mecanismo que explica este efecto parece ser la estimulación del apetito, mediante la interacción de estos medicamentos con diversos receptores cerebrales.

Existe una marcada diferencia en el riesgo de aumento de peso entre diferentes antipsicóticos, sin embargo, ningún agente debería ser considera- do como neutral, ya que la proporción de individuos que experimentan más de $7 \%$ de aumento de peso es mayor con cualquier antipsicótico atípico comparado con placebo ${ }^{13}$.

Es importante considerar que existen factores demográficos y características clínicas que se traducen en una mayor propensión al aumento de peso asociado al consumo de antipsicóticos. En particular, existe evidencia que sugiere que los niños y adolescentes que toman medicación antipsicótica tienen un mayor riesgo de aumento de peso y efectos metabólicos en comparación a los adultos que usan las mismas drogas ${ }^{12,14}$.

\section{Síndrome metabólico}

El síndrome metabólico (SM) es un conjunto de condiciones, incluyendo intolerancia a la glucosa, hipertensión arterial (HTA) y dislipidemia, que se asocian a un aumento en la probabilidad de sufrir enfermedades cardiovasculares.

Los pacientes con primer episodio psicótico, sin tratamiento antipsicótico previo, poseen una composición corporal alterada, con un depósito aumentado de grasa intraabdominal mientras que la grasa subcutánea se encuentra disminuida, lo cual ha surgido como un factor independiente para el desarrollo de SM y enfermedad cardiovascular $^{12}$.

Los antipsicóticos atípicos pueden inhibir la recaptación de glucosa en las células esqueléticas y hepáticas a través de la inhibición del transportador de la glucosa ${ }^{15}$. Además, estos pacientes presentan desde el inicio una alteración en la glicemia de ayuno, insulina basal y niveles de cortisol ${ }^{16}$. Estudios epidemiológicos señalan que los pacientes con esquizofrenia tienen, independiente del uso de antipsicóticos, mayor probabilidad de desarrollar algún grado de intolerancia a la glucosa incluyendo diabetes mellitus tipo 2 (DM2) ${ }^{17}$. En base a estos hallazgos es posible postular que las anormalidades metabólicas son una parte inherente de la enfermedad esquizofrénica.

Por otro lado, la HTA no se ha revelado como un factor ligado a la EQZ, sin embargo, el nivel de evidencia es bajo y se requiere mayor investigación para concluir si existe una relación directa entre estas enfermedades.

En cuanto a la dislipidemia, se ha encontrado una prevalencia de esta condición de entre $25 \%$ y $69 \%$ en los pacientes con EQZ. Estudios prospectivos muestran que el uso de antipsicóticos se asocia 
a un aumento del colesterol LDL y una disminución del colesterol HDL. También existe efecto de estos medicamentos sobre los triglicéridos, existiendo, en este caso, claras diferencias entre drogas: aquellos medicamentos que se asociaron a un mayor incremento de peso, como olanzapina y clozapina, también se asociaron a mayor aumento de triglicéridos plasmáticos ${ }^{12}$.

\section{Disrupción de ritmo circadiano}

Los pacientes con EQZ tienen característicamente insomnio de conciliación y mantenimiento, tanto si se encuentran en tratamiento antipsicóticos como si no, y sin relación con la estabilidad del ánimo, control de la enfermedad y funcionalidad diaria $^{18}$. Esta alteración se ha relacionado a vías dopaminérgicas y GABAérgicas, además de alteraciones de genes relacionados al ciclo circadiano como CLOCK y ARNTL y disfunción de la melatonina, tanto por disminución en su producción como por alteración en sus receptores ${ }^{19}$. Aunque existe evidencia limitada, la apnea obstructiva del sueño (AOS) se ha correlacionado con la EQZ. La AOS incrementa la morbilidad médica y psiquiátrica asociada con la EQZ. El diagnóstico y tratamiento de la AOS en EQZ puede mejorar la salud física general, la salud mental y la calidad de vida en estos pacientes tan vulnerables ${ }^{20}$.

En la población general, la deprivación de sueño y la desregulación de ritmo circadiano están asociados directamente al desarrollo de HTA, DM2 y otras alteraciones cardiometabólicas, por lo que se sospecha que estas condiciones pueden ser una causal importante en los trastornos de sueño de los pacientes con EQZ ${ }^{2}$. Esto acentúa la importancia de sospechar y tratar dichas condiciones en estos pacientes. Estudios preliminares muestran a la melatonina, en la EQZ, como un importante foco terapéutico, pero se requiere mayor investigación para garantizar su utilidad ${ }^{21}$.

\section{Cardiovascular}

La población con enfermedades mentales graves, como la esquizofrenia, tiene peor salud física y reducida expectativa de vida al compararla con la población general ${ }^{15}$. Se ha observado que la mortalidad por enfermedad cardíaca isquémica ha ido disminuyendo en la población general, sin embargo, se ha mantenido constante o aumentado en la población con desórdenes mentales, incluyendo las EQZ ${ }^{22}$. La evidencia indica que las patologías cardiovasculares constituyen la principal causa del aumento de mortalidad en la población con esquizofrenia ${ }^{3}$.

Es importante considerar que los pacientes con desórdenes mentales tienen una alta prevalencia de factores de riesgo modificables para enfermedad cardiovascular, incluyendo obesidad, HTA, diabetes mellitus y dislipidemia ${ }^{23}$.

Los pacientes con EQZ tienen un riesgo aumentado de muerte súbita ${ }^{24}$, lo cual ha sido atribuido principalmente al uso de antipsicóticos ${ }^{25}$, comúnmente, a los efectos bloqueadores de la repolarización cardíaca, representado por el incremento del segmento electrocardiográfico $\mathrm{QT}^{26,27}$.

Sin embargo, recientemente un estudio demostró que los pacientes con EQZ presentan una alta prevalencia del patrón electrocardiográfico del síndrome de Brugada (enfermedad hereditaria caracterizada por una anormalidad electrocardiográfica y un aumento del riesgo de muerte súbita), así como intervalos QT prolongados, lo cual no sería explicado por el uso de medicamentos. Estos patrones electrocardiográficos explicarían en parte el mayor riesgo de muerte súbita ${ }^{28}$.

Entonces podríamos plantear que la EQZ podría representar una patología con vulnerabilidad cardíaca específica a la muerte súbita ${ }^{29}$, lo cual es respaldado por un estudio de Mothi et al., que demuestra que la disfunción cardiovascular y metabólica está aumentada en parientes de primer grado de los pacientes con EQZ. Esto es sugerente de la existencia de un contexto genético común de las condiciones cardiometabólicas y los desórdenes psicóticos $^{30}$.

Los pacientes EQZ presentan también un mayor riesgo de infarto agudo al miocardio (IAM) que la población general. A pesar de los posibles efectos adversos cardiovasculares de los antipsicóticos, la asociación entre exposición prolongada al uso de antipsicóticos e IAM ha sido controversial, debido a la heterogeneidad en la metodología de los estudios ${ }^{31}$. Con respecto a la exposición temprana a antipsicóticos, un estudio reciente de $\mathrm{Wu}$ et al. propone que esta se asocia a riesgo aumentado de IAM ${ }^{3}$. Sin embargo, es importante considerar que previamente se ha demostrado que el uso de antipsicóticos disminuye la mortalidad en pacientes con enfermedad mental severa, por lo cual los beneficios del uso de antipsicóticos muy probablemente superan al riesgo de incremento de IAM $^{3}$. 


\section{Discapacidad cognitiva y riesgos cardiometabólicos}

La EQZ tiene efectos perjudiciales en la calidad de vida; principalmente porque los síntomas, en este caso cognitivos, comienzan en una edad temprana, y su recuperación completa se ha logrado parcialmente con las terapias actuales. Específicamente, la EQZ se caracteriza por múltiples déficits cognitivos, incluyendo principalmente atención, memoria y funciones ejecutivas. La mayoría de los problemas psicosociales, por ejemplo, dificultades en completar sus estudios o mantener su estabilidad laboral que determinan pobreza y exclusión social, son asociados con esta deficiencia cognitiva $^{32}$.

Por lo descrito, es de vital importancia discutir que la incidencia de la enfermedad cardiovascular y el síndrome metabólico se ha incrementado drásticamente en adultos, adolescente y niños en las últimas décadas. La hipertensión arterial, el índice de masa corporal superior al rango normal, la elevación del nivel de colesterol, la circunferencia de la cintura superior al normal, la diabetes mellitus, entre otros, se constituyen como los principales factores de riesgo de enfermedad cardiovascular y síndrome metabólico ${ }^{33}$. Además como factores de riesgo agregado para el desarrollo del síndrome metabólico, se suman el tabaquismo, alcoholismo, uso y abuso de drogas, dieta inadecuada y falta de ejercicio ${ }^{34}$. Últimamente, surge el interés del efecto que los factores de riesgo cardiometabólicos pueden tener sobre el rendimiento cognitivo de las personas, principalmente en EQZ.

Existe un interesante estudio exploratorio de la relación entre factores de riesgo cardiometabólico y el rendimiento cognitivo/académico en adolescentes sanos. Por ejemplo, se encontraron correlaciones entre la presión arterial y el desempeño académico. Los autores mencionan otros estudios en que se encontró que tanto la hipertensión, como la hipotensión juegan un papel determinante en el desarrollo y la progresión de deterioro cognitivo y demencia. Personas con hipertensión dan cuenta de rendimientos deficientes en tareas de atención, aprendizaje y memoria, funciones ejecutivas, habilidades visoespaciales, habilidades psicomotoras y de percepción. Por otro lado, personas con hipotensión tuvieron un rendimiento deficiente en tareas de memoria verbal a corto plazo, cálculo mental, función ejecutiva, atención selectiva y reconocimiento verbal, ambos grupos comparados con población normotensa ${ }^{33}$. Los pacientes con diabetes mellitus poseen un perfil neuropsicológico de deterioro cognitivo con un patrón de disfunción fronto-subcortical. En la DM2 los dominios más afectados son la atención, función ejecutiva, memoria verbal y velocidad de procesamiento de la información, manteniéndose conservada las funciones visoespaciales y el lenguaje $\mathrm{e}^{34}$.

La obesidad y el bajo peso también se han asociado con un rendimiento cognitivo deficiente, lo que aumenta el riesgo de desarrollar un cuadro demenciante. Con respecto a la hiperlipidemia existen resultados contradictorios, sin embargo, hay cierta evidencia que refiere la existencia de una relación entre los niveles totales de colesterol y un rendimiento deficiente en las medidas cognitivas realizadas, llegándose a la conclusión de que niveles alto de colesterol serían un importante factor de riesgo para el desarrollo de deterioro cognitivo y demencia ${ }^{33}$. Lo descrito, aunque preliminar aún, podría agravar el ya existente déficit cognitivo de los pacientes con EQZ, siendo muchos de ellos factores modificables clínicamente.

\section{Osteoporosis}

La osteoporosis es una enfermedad sistémica del esqueleto caracterizada por disminución de masa y deterioro de la microarquitectura del tejido óseo, con aumento de la fragilidad y mayor susceptibilidad a las fracturas ${ }^{35}$. Esta patología es un problema de gran importancia a nivel mundial ${ }^{36}$.

Al compararlos con la población normal, los pacientes con EQZ presentan una prevalencia mayor de osteoporosis y fracturas óseas, así como una densidad mineral ósea marcadamente disminuida $^{35,36}$. Los factores de riesgo para osteoporosis que se han identificado en esta población se pueden agrupar en dos tipos: modificables y no modificables. Los factores no modificables incluyen edad avanzada, raza asiática o caucásica e historia familiar de osteoporosis. En cuanto al sexo existen resultados controversiales en diversos estudios con respecto a pacientes que sufren de EQZ ${ }^{36}$.

Los factores modificables incluyen el IMC, tabaquismo, inactividad física, baja ingesta de calcio en dieta, deficiencia de vitamina $\mathrm{D}$, ciertos medicamentos antipsicóticos, consumo de café y alcohol ${ }^{37,38}$. Todas ellas relacionadas a malos há- 
bitos que poseen los pacientes con EQZ y tratados más profundamente en el apartado de hábitos. El comportamiento asociado a síntomas negativos podría influir en la poca actividad física y menor tendencia a salir, influyendo en la producción de vitamina $\mathrm{D}^{39}$.

Es importante considerar que la disminución de la densidad mineral ósea y la osteoporosis en EQZ se aprecian particularmente en pacientes en terapia de medicación antipsicótica ${ }^{40}$. Los mecanismos de osteoporosis inducida por antipsicóticos son complejos, pero probablemente el de mayor importancia es la hiperprolactinemia, causada por la mayoría de los antipsicóticos, aunque siendo este evento más marcado con el uso de risperidona, paliperidona y amisulpiride. El mecanismo de hiperprolactinemia sería el bloqueo del receptor dopaminérgico D2 en el lactótrofo, lo que anula la función inhibitoria de la dopamina sobre la secreción de prolactina ${ }^{36}$.

La hiperprolactinemia inducida por antipsicóticos puede afectar el metabolismo óseo de dos maneras: afecta directamente el recambio óseo al estimular la reabsorción ósea y la hiperprolactinemia prolongada puede causar hipogonadismo hipogonadotropo, que supone una disminución en la secreción de hormonas sexuales, lo cual influye en el metabolismo óseo (el hipoestrogenismo aumenta el riesgo de osteoporosis, y los bajos niveles de testosterona se asocian a osteopenia y osteoporosis) $^{36}$.

Al respecto de esta situación se ha propuesto estimular una dieta balanceada, suspender tabaquismo y disminuir la ingesta de cafeína y alcohol, además de iniciar tratamiento con vitamina $\mathrm{D}$. Junto a lo anterior se recomienda realizar evaluación de prolactinemia y, en caso de estar elevada, descartar otras causas y considerar cambiar el tratamiento antipsicótico o adicionar un agonista dopaminérgico, como el aripiprazol ${ }^{41}$.

\section{Enfermedad renal crónica}

Se ha evidenciado que los pacientes con EQZ tienen $25 \%$ más de probabilidad de desarrollar enfermedad renal crónica (ERC) que la población general, independiente de tratamiento antipsicótico y las comorbilidades médicas (DM2, HTA), por lo que se postula como un evento propio de la EQZ; sin embargo, la causa de esta relación no se conoce. Se postula que es la misma disfunción endotelial que predispone a los pacientes a de- sarrollar otras enfermedades como DM2 y HTA la que estaría causando la ERC, sin embargo, se necesita más investigación en el área. Se sugiere entonces buscar dirigidamente ERC en los pacientes con EQZ y manejar las comorbilidades para prevenirla ${ }^{42}$.

\section{Conclusión}

La EQZ es una enfermedad con un compromiso sistémico per se, más allá de lo que se describe clásicamente. La nueva información disponible obliga a un cambio de enfoque en el estudio y en el manejo de los pacientes con EQZ, destacando la necesidad de prevención cardiometabólica y de tamizaje de las enfermedades anteriormente descritas en todo paciente diagnosticado de EQZ, junto con un equipo multidisciplinario donde además del psiquiatra participe un cardiólogo, diabetólogo, nutricionista, entre otros.

Los estudios de seguimientos prolongados de pacientes esquizofrénicos para analizar cómo varían sus perfiles cardiovasculares, lipídicos, etc. en el tiempo, y de qué modo impacta el uso de antipsicóticos en ellos aún no se publican a la fecha de esta revisión.

Las investigaciones del impacto de estrategias de intervención en los factores de riesgo modificables está en una fase inicial, por ejemplo: Bobes et al. $2010^{7}$ encontraron, en comparación con la población general, que los pacientes con esquizofrenia mostraron mayor prevalencia de consumo de tabaco. Los pacientes fumadores que lo dejaron se beneficiarían con una reducción en la probabilidad de riesgo de algún evento cardiovascular. Otro ejemplo es descrito por Bartels y Desilets en $2012^{11}$ y concluyen que ciertas intervenciones en el estilo de vida (ejercicio, nutrición) parecen lograr pérdida de peso clínicamente significativa en pacientes con sobrepeso por enfermedad mental grave.

Finalmente, el conocimiento actual permite entender de mejor forma la EQZ, sin embargo, establece dos líneas en las que es necesario seguir investigando, en primer lugar: ¿hasta dónde alcanza el compromiso sistémico de la EQZ? En segundo lugar y más relevante aún: ¿cuál es el origen de estas enfermedades asociadas a la EQZ y cómo el compromiso sistémico está relacionado con el compromiso de la mente? 


\section{Referencias}

1. Fischer B, Buchanan R. Schizophrenia: Clinical manifestations, course, assessment, and diagnosis, web: UpToDate.com, Section Editor: Stephen Marder, MD, Deputy Editor: Richard Hermann, MD. Wolters Kluwer Health (Filadelfia). (http://www.uptodate.com/contents/ schizophrenia-clinical-manifestations-course-assessment-and-diagnosis) (Consultado en diciembre de 2015).

2. Robillard R, Rogers N, Whitwell B, Lambert T. Are Cardiometabolic and Endocrine Abnormalities Linked to Sleep Difficulties in Schizophrenia? A Hypothesis Driven Review. Clin Psychopharmacol Neurosci 2012; 10 (1): 1-12.

3. Wu S-I, Kao K-L, Chen S-C, Juang JJM, Lin C-J, Fang $\mathrm{C}-\mathrm{K}$, et al. Antipsychotic exposure prior to acute myocardial infarction in patients with serious mental illness. Acta Psychiatr Scand 2015; 131 (3): 213-22.

4. Correll CU, Robinson DG, Schooler NR. Cardiometabolic risk in patients with first-episode schizophrenia spectrum disorders: baseline results from the RAISE-ETP study. JAMA Psychiatry 2014; 71 (12): 1350-63.

5. Kisely S, Smith M. Mortality in individuals who have had psychiatric treatment: population-based study in Nova Scotia. Br J Psychiatry 2005; 187: 552-8.

6. Newcomer JW. Antipsychotic medications: metabolic and cardiovascular risk. J Clin Psychiatry 2007; 68 Suppl 4: 8-13.

7. Bobes J, Arango C, García-García M, Rejas J. Healthy lifestyle habits and 10-year cardiovascular risk in schizophrenia spectrum disorders: An analysis of the impact of smoking tobacco in the CLAMORS schizophrenia cohort. Schizoph Res 2010; 119: 101-9.

8. Roick C, Fritz-Wieacker A, Matschinger H. Health habits of patients with schizophrenia. Soc Psychiatry Psychiatr Epidemiol April 2007; 42 (4): 268-76.

9. Lavin MR, Siris SG, Mason SE. What Is the Clinical Importance of Cigarette Smoking in Schizophrenia? Am J Addict 2010; 5 (3): 189-208.

10. Volkow ND. Substance Use Disorders in Schizophrenia-Clinical Implications of Comorbidity. Schizophr Bull 2009; 35 (3): 469-72.

11. Bartels S, Desilets R. Health Promotion Programs for People with Serious Mental Illness (Prepared by the Dartmouth Health Promotion Research Team). Washington, D.C. SAMHSA-HRSA Center for Integrated Health Solutions. January 2012. (http://www.integration. samhsa.gov/Health_Promotion_White_Paper_Bartels_ Final_Document.pdf) (Consultado en marzo de 2016).

12. De Hert M, Dekker J, Wood D, Kahl K, Holt R, Möller
H. Cardiovascular disease and diabetes in people with severe mental illness position statement from the European Psychiatric Association (EPA), supported by the European Association for the Study of Diabetes (EASD) and the European Society of Cardiology (ESC). Eur Psychiatry 2009; 24 (6): 412-24.

13. Citrome L. Risk-Benefit Analysis of Available Treatments for Schizophrenia. Psychiatric Times 2007; 1: 27-30.

14. Cohen D, Huinink S. Atypical antipsychotic-induced diabetes mellitus in child and adolescent psychiatry. CNS Drugs 2007; 21 (12): 1035-8.

15. Starrenburg F, Bogers J. How can antipsychotics cause Diabetes Mellitus? Insights based on receptor-binding profiles, humoral factors and transporter proteins. Eur Psychiatry 2009; 24 (3): 164-70.

16. Ryan MC, Collins $\mathrm{P}$, Thakore JH. Impaired fasting glucose tolerance in first-episode, drug-naive patients with schizophrenia. Am J Psychiatry 2003; 160 (2): 284-9.

17. Bushe C, Holt R. Prevalence of diabetes and impaired glucose tolerance in patients with schizophrenia. Br J Psychiatry 2004; 184 (47): 567-71.

18. Wulff K, Dijk DJ, Middleton B, Foster R, Joyce EM. Sleep and circadian rhythm disruption in schizophrenia. Br J Psychiatry 2012; 200 (4): 308-16.

19. Monti J, BaHammam A, Pandi-Perumal S, Bromundt V, Spence D, Cardinali D, et al. Sleep and circadian rhythm dysregulation in schizophrenia. Prog Neuropsychopharmacol Biol Psychiatry 2013; 43: 209-16.

20. Kalucy M, Grunstein, R, Lambert T, Glozier N. Obstructive sleep apnoea and schizophrenia-A research agenda. Sleep Med Rev 2013; 17 (5): 357-65.

21. Shamir E, Laudon M, Barak Y, Anis Y, Rotenberg V, Elizur A, et al. Melatonin improves sleep quality of patients with chronic schizophrenia. J Clin Psychiatry 2000; 61 (5): 373-7.

22. Lawrence D, Holman C, Jablensky A, Hobbs M. Death rate from ischaemic heart disease in Western Australian psychiatric patients 1980-1998. Br J Psychiatry 2003; 182: 31-6.

23. Khasawneh F, Shankar G. Minimizing Cardiovascular Adverse Effects of Atypical Antipsychotic Drugs in Patients with Schizophrenia. Cardiol Res and Pract; vol 2014; 2014: 273060. (http://dx.doi.org/10.1155/2014/ 273060).

24. Appleby L, Thomas S, Ferrier N, Lewis G, Shaw J, Amos T. Sudden unexplained death in psychiatric in-patients. Br J Psychiatry 2000; 176: 405-6.

25. Timour Q, Frassati D, Descotes J, Chevalier P, Christé G, Chahine M. Sudden death of cardiac origin and psychotropic drugs. Front Pharmacol 2012; 3: 76. 
26. Ray WA, Chung CP, Murray KT, Hall K, Stein CM. Atypical antipsychotic drugs and the risk of sudden cardiac death. N Engl J Med 2009; 360: 225-35.

27. Haddad PM, Anderson IM. Antipsychotic-related QTc prolongation, torsade de pointes and sudden death. Drugs 2002; 62: 1649-71.

28. Blom M, Cohen D, Seldenrijk A, Penninx B, Nijpels G, Stehouwer C, et al. Brugada syndrome ECG is highly prevalent in schizophrenia. Circ Arrhythm Electrophysiol 2014; 7 (3): 384-91.

29. Beary M, Hodgson R, Wildgust H. A critical review of major mortality risk factors for all-cause mortality in first-episode schizophrenia: clinical and research implications. J Psychopharmacol 2012; 26: 52-61.

30. Mothi S, Tandon N, Padmanabhan J, Mathew I, Clementz B, Tamminga C, et al. Increased cardiometabolic dysfunction in first-degree relatives of patients with psychotic disorders. Schizophr Res 2015; 165 (1): 103-7.

31. Brauer R, Douglas I, Smeeth L. The association between antipsychotic agents and the risk of myocardial infarction: a systematic review. Br J Clin Pharmacol 2011; 72: 871-8.

32. Orellana G, Peña M, Slachevsky A. Executive attention impairment in first episode Schizophrenia. BMC Psychiatric 2012; 12: 154.

33. Yeh T, Cho Y, Yeh T, Hu C, Lee L, Chang C. An Exploratory Analysis of the Relationship between Cardiometabolic Risk Factors and Cognitive/Academic Performance among Adolescents. Biomed Res Int. 2015;2015:520619. doi: 10.1155/2015/520619. Epub 2015 Jun 7.
34. Cervantes A, Callejas J, Rodríguez M. Función cognitiva y factores cardiometabólicos en diabetes mellitus tipo 2. Med Int Mex 2009; 25 (5): 386-92.

35. NIH. Consensus Development Panel on Osteoporosis Prevention. Diagnosis and Therapy. JAMA 2001; 285: 785-95.

36. Wu H, Deng L, Zhao L, Zhao J, Li L, Chen J. Osteoporosis associated with antipsychotic treatment in schizophrenia. Int J Endocrinol 2013; 2013: 167138. doi: 10.1155/2013/167138.

37. Howard L, Kirkwood G, and Leese M. Risk of hip fracture in patients with a history of schizophrenia. Br J Psychiatry 2007; 190: 129-34.

38. Cummings S, Melton L. Osteoporosis I: epidemiology and outcomes of osteoporotic fractures. Lancet 2002; 359 (9319): 1761-7.

39. Pekkinen M, Viljakainen H, Saarnio E, Lamberg-Allardt C, Makitie O. Vitamin D is a major determinant of bone mineral density at school age. PLoS One 2012; 7 (7): e40090. doi: 10.1371/journal.pone.0040090.

40. Pouwels S, Van Staa T, Egberts A, Leufkens H, Cooper C, de Vries F. Antipsychotic use and the risk of hip/ femur fracture: a population-based case-control study. Osteoporos Int 2009; 20 (9): 1499-506.

41. Kishimoto T, de Hert M, Carlson H, Manu P, Correll C. Osteoporosis and fracture risk in people with schizophrenia. Curr Opin Psychiatry 2012; 25 (5): 415-29.

42. Tzeng N, Hsu Y, Ho S, Kuo Y, Lee H, Yin Y, et al. Is schizophrenia associated with an increased risk of chronic kidney disease? A nationwide matched-cohort study. BMJ Open 2015; 5 (1): 1-8. 